PROCEEDINGS OF THE

AMERICAN MATHEMATICAL SOCIETY

Volume 138, Number 4, April 2010, Pages 1495-1499

S 0002-9939(09)10190-9

Article electronically published on December 8, 2009

\title{
NOTE ON BETA ELEMENTS IN HOMOTOPY, AND AN APPLICATION TO THE PRIME THREE CASE
}

\author{
KATSUMI SHIMOMURA
}

(Communicated by Brooke Shipley)

\begin{abstract}
Let $S_{(p)}^{0}$ denote the sphere spectrum localized at an odd prime $p$. Then we have the first beta element $\beta_{1} \in \pi_{2 p^{2}-2 p-2}\left(S_{(p)}^{0}\right)$, whose cofiber we denote by $W$. We also consider a generalized Smith-Toda spectrum $V_{r}$ characterized by $B P_{*}\left(V_{r}\right)=B P_{*} /\left(p, v_{1}^{r}\right)$. In this note, we show that an element of $\pi_{*}\left(V_{r} \wedge W\right)$ gives rise to a beta element of homotopy groups of spheres. As an application, we show the existence of $\beta_{9 t+3}$ at the prime three to complete a conjecture of Ravenel's: $\beta_{s} \in \pi_{16 s-6}\left(S_{(3)}^{0}\right)$ exists if and only if $s$ is not congruent to 4,7 or $8 \bmod 9$.
\end{abstract}

\section{IntRoduCtion AND STATEMENTS OF RESUlts}

Let $B P$ denote the Brown-Peterson spectrum at an odd prime number $p$. Then, we have the Hopf algebroid $B P_{*} B P$ over $B P_{*}$, where

$$
B P_{*}=\pi_{*}(B P)=\mathbb{Z}_{(p)}\left[v_{1}, v_{2}, \ldots\right] \quad \text { and } B P_{*} B P=B P_{*}(B P)=B P_{*}\left[t_{1}, t_{2}, \ldots\right]
$$

for $v_{i} \in B P_{2 p^{i}-2}$ and $t_{i} \in B P_{2 p^{i}-2} B P$. This gives rise to the Adams-Novikov spectral sequence converging to homotopy groups $\pi_{*}(X)$ of a connective spectrum $X$ with $E_{2}$-term

$$
E_{2}^{s, t}(X)=\operatorname{Ext}_{B P_{*} B P}^{s, t}\left(B P_{*}, B P_{*}(X)\right) .
$$

We consider the sphere spectrum $S^{0}$, the modulo $p$ Moore spectrum $M$ and a cofiber $V_{r}$ of the map $\alpha^{r}: \Sigma^{r q} M \rightarrow M$ for a positive integer $r$ and the Adams map $\alpha: \Sigma^{q} M \rightarrow M$, so that

$$
S^{0} \stackrel{p}{\longrightarrow} S^{0} \stackrel{i}{\longrightarrow} M \stackrel{j}{\longrightarrow} S^{1} \text { and } \quad \Sigma^{r q} M \stackrel{\alpha^{r}}{\longrightarrow} M \stackrel{i_{r}}{\longrightarrow} V_{r} \stackrel{j_{r}}{\longrightarrow} \Sigma^{r q+1} M
$$

are cofiber sequences. Here $q=2 p-2$ as usual. Suppose that $v_{1}^{t} v_{2}^{s} \in E_{2}^{0,(s p+s+t) q}\left(V_{r}\right)$ for integers $r>0, s>0$ and $t \geq 0$. Then, we define the beta element $\beta_{s / r-t}$ in the $E_{2}$-term by

$$
\beta_{s / r-t}=\delta \delta_{r}\left(v_{1}^{t} v_{2}^{s}\right) \in E_{2}^{2,(s p+s+t-r) q}\left(S^{0}\right)
$$

for the connecting homomorphisms $\delta_{r}: E_{2}^{s, t}\left(V_{r}\right) \rightarrow E_{2}^{s+1, t-r q}(M)$ and $\delta: E_{2}^{s, t}(M) \rightarrow$ $E_{2}^{s+1, t}\left(S^{0}\right)$ associated to the cofiber sequences (1.1). We note that if $r-t=r^{\prime}-t^{\prime}$, then $\beta_{s / r-t}=\beta_{s / r^{\prime}-t^{\prime}}$, and abbreviate $\beta_{s / 1}$ to $\beta_{s}$. In this paper, we study which

Received by the editors April 19, 2009.

2010 Mathematics Subject Classification. Primary 55Q45.

(C)2009 American Mathematical Society Reverts to public domain 28 years from publication 
of these elements survives to the homotopy groups $\pi_{*}\left(S^{0}\right)$ of spheres. For a prime greater than three, the following beta elements survive:

$\beta_{s}$ for $s>0$ by L. Smith 9 ,

$\beta_{t p / r}$ for $t>0$ and $r \leq p$ with $(t, r) \neq(1, p)$ by S. Oka [4, [5, and

$\beta_{t p^{2} / r}$ for $t>0$ and $r \leq 2 p$ by S. Oka $[6$.

At the prime three, $\beta_{s}$ survives if $s<4$ by Toda [10, and does not if $s=4$, and does if $s=5$ by Oka 2 . Ravenel then conjectured that $\beta_{s}$ survives in the spectral sequence if and only if $s \equiv 0,1,2,3,5,6 \bmod 9$. In [8], we proved the 'only if' part. For the survivors, we have

$\beta_{s}$ for $s>0$ with $s \equiv 0,1,2,5,6 \bmod 9$ by M. Behrens and S. Pemmaraju [1].

We note that the element $\beta_{1} \in \pi_{p q-2}\left(S^{0}\right)$ is given by

$$
\beta_{1}=j j_{1}\left[\beta i_{1}\right] i
$$

for the maps $i, j, i_{1}$ and $j_{1}$ are the maps given in (1.1). Here, $\left[\beta i_{1}\right]$ denotes $\beta i_{1}$ for the self-map $\beta \in[V(1), V(1)]_{(p+1) q}\left(V(1)=V_{1}\right)$ constructed by Smith [9] at a prime greater than three, and the generator of the homotopy group $[M, V(1)]_{16}$ given in [11] at the prime three. We define $W$ by the cofiber sequence

$$
S^{p q-2} \stackrel{\beta_{1}}{\longrightarrow} S^{0} \stackrel{\iota}{\longrightarrow} W \stackrel{\kappa}{\longrightarrow} S^{p q-1} .
$$

Then we have our main theorem:

Theorem 1.4. Suppose that there is an element $B_{s} \in \pi_{s(p+1) q}\left(V_{r} \wedge W\right)$ detected by $v_{2}^{s} \in E_{2}^{0, s(p+1) q}\left(V_{r} \wedge W\right)$. Then, the beta element $\beta_{s / l}$ for $0<l \leq r-2$ survives to $\pi_{(s p+s-l) q-2}\left(S^{0}\right)$.

As an example, we have

Lemma 1.5. At an odd prime, there exists $B_{t p} \in \pi_{t p(p+1) q}\left(V_{p} \wedge W\right)$ for $t>0$ detected by $v_{2}^{t p} \in E_{2}^{0, t p(p+1) q}\left(V_{p} \wedge W\right)$.

Corollary 1.6. The beta elements $\beta_{t p / l}$ for $t>0$ and $0<l \leq p-2$ survive.

This corollary shows that $\beta_{3 t}$ survives at the prime three and completes a proof of the conjecture.

\section{Proofs of Results}

Applying the $B P_{*}$-homology to the first cofiber sequence of (1.1), we obtain

$$
B P_{*}(M)=B P_{*} /(p) \text {. }
$$

We observe the $E_{2}$-term $E_{2}^{*}(X)$ of the Adams-Novikov spectral sequence as the cohomology of the reduced cobar complex $\widetilde{\Omega}_{B P_{*} B P} B P_{*}(X)$. Then, we have a vanishing line for a (-1)-connected spectrum $X$ :

$$
E_{2}^{s, t}(X)=0 \quad \text { if } t<s q .
$$

The structure maps of the Hopf algebroid $B P_{*} B P$ act on generators by

$$
\begin{aligned}
\eta_{R}\left(v_{1}\right) & =v_{1}+p t_{1} \\
\eta_{R}\left(v_{2}\right) & \equiv v_{2}+v_{1} t_{1}^{p}-v_{1}^{p} t_{1} \quad \bmod (p), \quad \text { and } \\
\Delta\left(t_{1}\right) & =t_{1} \otimes 1+1 \otimes t_{1}
\end{aligned}
$$


(cf. [7]). By this, we see that $v_{1} \in E_{2}^{0, q}(M)$. Since $E_{2}^{s q+1,(s+1) q}(M)=0$ for $s>0$ by (2.1), $v_{1}$ is a permanent cycle. The Adams map $\alpha$ in (1.1) is given by $\alpha=m\left(M \wedge v_{1}\right)$ for the multiplication $m$ of $M$, and so it induces $v_{1}$-multiplication on the $B P_{*}$-homology. It follows that

$$
B P_{*}\left(V_{r}\right)=B P_{*} /\left(p, v_{1}^{r}\right)
$$

for $r>0$, and we see that

$$
v_{1} \in E_{2}^{0, q}\left(V_{r}\right) \quad \text { and } \quad v_{2}^{p} \in E_{2}^{0,\left(p^{2}+p\right) q}\left(V_{p}\right)
$$

by (2.2). For later use, we notice that

$$
\alpha i=m\left(i \wedge v_{1}\right)=v_{1} .
$$

From [12, we get

Lemma 2.5. Suppose that $t-s<\left(p^{2}+p+1\right) q$. In this range, the Adams-Novikov $E_{2}$-term $E_{2}^{*, *}(M)$ is a subquotient of $\mathbb{Z} / p\left[v_{1}, v_{2}\right] \otimes\left\{h_{0}, h_{1}, h_{2}, g_{0}, k_{0}, k_{0} h_{0}, h_{0} h_{2}\right\} \otimes$ $P\left(b_{0}, b_{1}, b_{20}\right)$. Here the bi-degrees of the generators are:

$$
\begin{aligned}
& \left|h_{i}\right|=\left(1, p^{i} q\right)(i=0,1,2), \quad\left|g_{0}\right|=(2,(p+2) q), \quad\left|k_{0}\right|=(2,(2 p+1) q), \\
& \left|b_{0}\right|=(2, p q), \quad\left|b_{1}\right|=\left(2, p^{2} q\right), \quad \text { and }\left|b_{20}\right|=\left(2,\left(p^{2}+p\right) q\right) .
\end{aligned}
$$

Proof. We have short exact sequences $0 \longrightarrow B P_{*} /(p) \stackrel{v_{1}}{\longrightarrow} B P_{*} /(p) \longrightarrow B P_{*} /\left(p, v_{1}\right)$ $\longrightarrow 0$ and $0 \longrightarrow B P_{*} /\left(p, v_{1}\right) \stackrel{v_{2}}{\longrightarrow} B P_{*} /\left(p, v_{1}\right) \longrightarrow B P_{*} /\left(p, v_{1}, v_{2}\right) \longrightarrow 0$, which give rise to Bockstein spectral sequences converging to the Adams-Novikov $E_{2}$-terms $E_{2}^{*}(M)$ and $E_{2}^{*}\left(V_{1}\right)$ with $E_{1}$-terms $E_{2}^{*}\left(V_{1}\right)$ and $\operatorname{Ext}_{B P_{*} B P}^{*}\left(B P_{*}, B P_{*} /\left(p, v_{1}, v_{2}\right)\right)$, respectively. In our range, we have $\operatorname{Ext}_{B P_{*} B P}^{*}\left(B P_{*}, B P_{*}^{*} /\left(p, v_{1}, v_{2}\right)\right)=\operatorname{Ext}_{\mathcal{P}}^{*}(\mathbb{Z} / p, \mathbb{Z} / p)$ for the subalgebra $\mathcal{P}$ of the Steenrod algebra generated by the reduced power operations. Thus, $E_{2}^{*}(M)$ is a subquotient of $\mathbb{Z} / p\left[v_{1}, v_{2}\right] \otimes \operatorname{Ext}_{\mathcal{P}}^{*}(\mathbb{Z} / p, \mathbb{Z} / p)$. We now get the structure of $\operatorname{Ext}_{\mathcal{P}}^{*}(\mathbb{Z} / p, \mathbb{Z} / p)$ from $[12$.

Corollary 2.6. In our range, we have a vanishing line: $E_{2}^{2 s+\varepsilon, t q}(V)=0$ for $V=M, V_{r}$, if $t<p s+\varepsilon$. Here, $\varepsilon=0,1$.

Lemma 2.7. Let $\delta: E_{2}^{s}(M) \rightarrow E_{2}^{s+1}\left(S^{0}\right)$ be the connecting homomorphism associated with the first cofiber sequence in (1.1). Then, it is a derivation and

$$
\delta\left(v_{1}\right)=h_{0}, \quad \delta\left(h_{2}\right)=-b_{1} \quad \text { and } \quad \delta\left(b_{0}\right)=0 .
$$

Proof. Note that $h_{i}$ and $b_{i}$ are represented by cocycles $t_{1}^{p^{i}}$ and $\sum_{k=1}^{p-1} \frac{1}{p}\left(\begin{array}{l}p \\ k\end{array}\right) t_{1}^{p-k} \otimes t_{1}^{k}$ of the cobar complex. By (2.2), we see that the differential $d$ of the cobar complex acts on $v_{1}$ and $t_{1}^{p^{i}}$ as $d\left(v_{1}\right)=p t_{1}$ and $d\left(t_{1}^{p^{i}}\right)=-p b_{i-1}$ for $i>0$. The lemma now follows from the definition of the connecting homomorphism.

The cofiber sequence (1.3) induces a split short exact sequence

$$
0 \longrightarrow E_{2}^{s . t}(V) \stackrel{\iota_{*}}{\longrightarrow} E_{2}^{s, t}(V \wedge W) \stackrel{\kappa_{*}}{\longrightarrow} E_{2}^{s, t-p q+1}(V) \longrightarrow 0
$$

of $E_{2}$-terms for $V=M$ and $V_{r}$, and so

$$
E_{2}^{*}(V \wedge W)=E_{2}(V) \oplus g E_{2}(V),
$$

where $g$ denotes a generator of degree $p q-1$ such that $\kappa_{*}(x g)=x$. Since the $E_{3}$-term is a homology of $E_{2}$-terms and $d_{2}(g)=\beta_{1}$ for the element $\beta_{1}$ in (1.2), we have the long exact sequence

$$
E_{3}^{s, t}(M) \stackrel{\partial}{\longrightarrow} E_{3}^{s+2, t+p q}(M) \stackrel{\iota_{*}}{\longrightarrow} E_{3}^{s+2, t+p q}(M \wedge W) \stackrel{\kappa_{*}}{\longrightarrow} E_{3}^{s+2, t+1}(M)
$$


with the connecting homomorphism $\partial$ given by $\partial(x)=x \beta_{1}$.

Lemma 2.9. The element $v_{2}^{p} \in E_{2}^{0}\left(V_{p} \wedge W\right)$ in (2.3) survives to an element $B_{p} \in$ $\pi_{p(p+1) q}\left(V_{p} \wedge W\right)$.

Proof. Consider the cofiber sequence (1.1) with $r=p$. In the Adams-Novikov spectral sequence for computing $\pi_{*}\left(S^{0}\right)$, we have the Toda differential $d_{q+1}\left(b_{1}\right)=$ $h_{0} b_{0}^{p} \in E_{2}^{q+3,\left(p^{2}+1\right) q}\left(S^{0}\right)$ up to a nonzero scalar. By Lemma 2.5. $E_{2}^{q+2,\left(p^{2}+1\right) q}(M)$ is a subquotient of $\left\{v_{1} b_{0}^{p}\right\}$. Since $\delta\left(v_{1} b_{0}^{p}\right)=h_{0} b_{0}^{p}$, we see that $d_{q+1}\left(h_{2}\right)=v_{1} b_{0}^{p} \in$ $E_{2}^{q+2,\left(p^{2}+1\right) q}(M)$ up to a nonzero scalar by Lemma 2.7. Note that $\beta_{1}=b_{0}$. In the exact sequence (2.8), $v_{1} b_{0}^{p}=\partial\left(v_{1} b_{0}^{p-1}\right)$, and so $d_{q+1}\left(\iota_{*}\left(h_{2}\right)\right)=0$ in $E_{3}^{q+2,\left(p^{2}+1\right) q}(M \wedge$ $W)$. Besides, Corollary 2.6 shows that $E_{2}^{s q+2,\left(p^{2}+s\right) q}(M)=0$ for $s>1$, and we see that $\iota_{*}\left(h_{2}\right) \in E_{2}^{1, p^{2} q}(M \wedge W)$ is a permanent cycle, which detects an element $\beta_{p / p}^{\prime} \in \pi_{p^{2} q-1}(M \wedge W)$. Send it by $\alpha^{p}$ in (1.1). The element $\alpha^{p} \beta_{p / p}^{\prime} \in$ $\pi_{\left(p^{2}+p\right) q-1}(M \wedge W)$ is detected by an element of $E_{2}^{q+1,\left(p^{2}+p+1\right) q}(M \wedge W)$, since the $E_{2}$-term $E_{2}^{s q+1, p(p+1) q+s q}(M \wedge W)=E_{2}^{s q+1, p(p+1) q+s q}(M)$ for $s>1$ is zero by Corollary 2.6. The $E_{2}$-term $E_{2}^{q+1,\left(p^{2}+p+1\right) q}(M)$ for $s=1$ is a subquotient of

$$
h_{0} b_{0} b_{1}(p=3), v_{1}^{p-1} v_{2} h_{0} b_{0}^{p-1}, v_{1}^{2 p} h_{0} b_{0}^{p-1}, v_{2} h_{1} b_{0}^{p-1}, v_{1}^{p+1} h_{1} b_{0}^{p-1}, v_{1}^{p-1} k_{0} h_{0} b_{0}^{p-2}
$$

by Lemma 2.5. and so the $E_{3}$-term $E_{3}^{q+1,\left(p^{2}+p+1\right) q}(M \wedge W)=0$ by (2.8). Therefore, $\alpha^{p} \beta_{p / p}^{\prime}=0$ and $\beta_{p / p}^{\prime}$ is pulled back to an element $B_{p}$ under the map $j_{p}$.

We call a spectrum $R$ a ring spectrum if there exist a multiplication $\mu: R \wedge R \rightarrow R$ and a unit $\iota: S^{0} \rightarrow R$ such that $\mu(\iota \wedge R)=1_{R}=\mu(R \wedge \iota): R \rightarrow R$. By [3, Ex. 2.9] and [3, Ex. 5.7], we have

$$
W \text { and } V_{r} \text { for } r>1 \text { are ring spectra. }
$$

In particular, the spectrum $R_{r}=V_{r} \wedge W$ for $r>1$ is a ring spectrum with multiplication $m_{r}=\left(\mu_{r} \wedge \mu_{W}\right)\left(V_{r} \wedge T \wedge W\right): R_{r} \wedge R_{r}=V_{r} \wedge W \wedge V_{r} \wedge W \rightarrow$ $V_{r} \wedge V_{r} \wedge W \wedge W \rightarrow V_{r} \wedge W=R_{r}$, where $T$ denotes the switching map and $\mu_{r}$ and $\mu_{W}$ are the multiplications of $V_{r}$ and $W$, respectively.

Proof of Lemma 1.5. Since $R_{p}=V_{p} \wedge W$ is a ring spectrum, we obtain a self$\operatorname{map}\left[\beta^{p}\right]: R_{p} \stackrel{R_{p} \wedge B_{p}}{\longrightarrow} R_{p} \wedge R_{p} \stackrel{m_{p}}{\longrightarrow} R_{p}$ inducing $v_{2}^{p}$ on $B P_{*}$-homology. Now put $B_{t p}=\left[\beta^{p}\right]^{t-1} B_{p}$ to see the lemma.

We consider the element $i_{r} \alpha^{2} i \in \pi_{2 q}\left(V_{r}\right) \cong \pi_{2 q}(M)=\mathbb{Z} / p\left\{\alpha^{2} i\right\}$ for $r>2$ and for the maps in (1.1), which is detected by the element $v_{1}^{2} \in E_{2}^{0}\left(V_{r}\right)$ by (2.4).

Lemma 2.11. Let $r>2$. There exists an element $\eta_{r} \in \pi_{(p+2) q-1}\left(V_{r} \wedge W\right)$ such that $\kappa_{*}\left(\eta_{r}\right)=i_{r} \alpha^{2} i \in \pi_{2 q}\left(V_{r}\right)$. Besides, it is detected by $v_{1}^{2} g \in E_{2}^{0}\left(V_{r} \wedge W\right)=$ $E_{2}^{0}\left(V_{r}\right) \oplus g E_{2}^{0}\left(V_{r}\right)$.

Proof. Putting $\delta=i j$ for the maps $i, j$ in (1.1), we have Yamamoto's relation $\alpha^{2} \delta=2 \alpha \delta \alpha-\delta \alpha^{2} \in[M, M]_{2 q-1}$ (cf. [11]). We compute

$$
\alpha^{2} i \beta_{1}=\alpha^{2} \delta j_{1}\left[\beta i_{1}\right] i=\left(\delta \alpha^{2}+\alpha \delta \alpha\right) j_{1}\left[\beta i_{1}\right] i=0,
$$

since $\alpha j_{1}=0$ by (1.1). It follows that $i_{r} \alpha^{2} i \in \pi_{2 q}\left(V_{r}\right)$ is pulled back to an element $\eta_{r} \in \pi_{(p+2) q-1}\left(V_{r} \wedge W\right)$ as desired. Since $i_{r} \alpha^{2} i$ is detected by $v_{1}^{2} \in E_{2}^{0}\left(V_{r}\right), \eta_{r}$ is detected by the element $v_{1}^{2} g \in E_{2}^{0}\left(V_{r} \wedge W\right)=E_{2}^{0}\left(V_{r}\right) \oplus g E_{2}^{0}\left(V_{r}\right)$. 
Proof of Theorem 1.4. Consider the product $B_{s} \eta_{r} \in \pi_{*}\left(V_{r} \wedge W\right)$ for the element $\eta_{r}$ in Lemma 2.11. Then, it is detected by $v_{1}^{2} v_{2}^{s} g$, since $\eta_{r}$ induces a $B P_{*} B P$-comodule map $\left(\eta_{r}\right)_{*}: B P_{*}\left(V_{r}\right) \rightarrow B P_{*}\left(V_{r} \wedge W\right)$ such that $\left(\eta_{r}\right)_{*}(x)=v_{1}^{2} x g$ and $B_{s}$ is detected by $v_{2}^{s}$. The map $\kappa_{*}: E_{2}^{0}\left(V_{r} \wedge W\right) \rightarrow E_{2}^{0}\left(V_{r}\right)$ assigns $v_{1}^{2} v_{2}^{s} g$ to $v_{1}^{2} v_{2}^{s}$, which is a permanent cycle detected by $\kappa\left(B_{s} \eta_{r}\right)$. Now putting $\beta_{s / l}=j j_{l+2} a^{r, l+2} \kappa\left(B_{s} \eta_{r}\right)=$ $j \alpha^{r-2-l} j_{r} \kappa\left(B_{s} \eta_{r}\right) \in \pi_{*}\left(S^{0}\right)$ for $l<r-2$, we see the theorem by the Geometric Boundary Theorem (cf. [7]). Here, $a^{r, k}$ denotes a map in the cofiber sequence $V_{r-k} \longrightarrow V_{r} \stackrel{a^{r, k}}{\longrightarrow} V_{k}$ obtained from applying the $3 \times 3$ Lemma to the cofiber sequences of (1.1) for $r-k, r$ and $k$. We note that it satisfies $j_{k} a^{r, k}=\alpha^{r-k} j_{r}$.

\section{REFERENCES}

1. M. Behrens and S. Pemmaraju, On the existence of the self map $v_{2}^{9}$ on the Smith-Toda complex $V(1)$ at the prime 3, Contemp. Math., 346, Amer. Math. Soc., Providence, RI, 2004, 9-49. MR2066495 (2005b:55029)

2. S. Oka, The homotopy groups of spheres. II, Hiroshima Math. J. 2 (1972), 99-161. MR0322865 $(48: 1226)$

3. S. Oka, Ring spectra with few cells, Japan J. Math. 5 (1979), 81-100. MR614695 (82i:55009)

4. S. Oka, A new family in the stable homotopy groups of sphere. I, Hiroshima Math. J. 5 (1975), 87-114. MR0380791 (52:1688)

5. S. Oka, A new family in the stable homotopy groups of sphere. II, Hiroshima Math. J. 6 (1976), 331-342. MR0418096 (54:6140)

6. S. Oka, Realizing some cyclic $B P_{*}$-modules and applications to stable homotopy of spheres, Hiroshima Math. J. 7 (1977), 427-447. MR0474290 (57:13937)

7. D. C. Ravenel, Complex Cobordism and Stable Homotopy Groups of Spheres, Second edition, AMS Chelsea Publishing, Amer. Math. Soc., Providence, RI, 2004. MR0860042 (87j:55003)

8. K. Shimomura, The homotopy groups of the $L_{2}$-localized Toda-Smith spectrum $V(1)$ at the prime 3, Trans. Amer. Math. Soc. 349 (1997), 1821-1850. MR.1370651 (97h:55010)

9. L. Smith, On realizing complex bordism modules. IV. Applications to the stable homotopy groups of spheres, Amer. J. Math. 99 (1977), 418-436. MR0433450 (55:6426)

10. H. Toda, p-primary components of homotopy groups. IV, Mem. Coll. Sci. Univ. Kyoto Ser. A Math. 32 (1959), 297-332. MR0111041 (22:1906)

11. H. Toda, Algebra of stable homotopy of $\mathbb{Z}_{p}$-spaces and applications, J. Math. Kyoto Univ. 11 (1971), 197-251. MR0293631 (45:2708)

12. H. Toda, On spectra realizing exterior parts of the Steenrod algebra, Topology 10 (1971), 53-65. MR0271933(42:6814)

Department of Mathematics, Faculty of Science, Kochi University, Kochi, 780-8520, JAPAN

E-mail address: katsumi@kochi-u.ac.jp 